\title{
On Human Malaria Parasite-Host Relationship, Sterile Immune Response and Cancer
}

\author{
Filiberto Malagón*, Jorge Gonzáles-Angúlo, Elba Carrasco and Norma Rivera \\ Department of Microbiology and Parasitology, National Autonomous University of Mexico, Mexico
}

Submission: December 21, 2017; Published: January 08, 2018

"Correspondence Address: Filiberto Malagón, Malariology Laboratory, Building A, 2nd floor, Faculty of Medicine, National Autonomous University of Mexico, 04510, Coyoacán, Mexico,Tel: 5255 56232465; Email: malagon@unam.mx

\begin{abstract}
Brief semblance on the story of research on malaria vaccine development through the time since the discovery of the malaria parasites to our days is presented, and emphasis on general results obtained is done. The emergence of a purified living sporozoite vaccine attenuated by radiation, and cryopreserved, that promise to control global morbidity and mortality of the malaria in endemic countries, providing sterile immunization against sporozoites of Plasmodium falciparum is commented. Experimental facts on sterile immune state and development of neoplasms when after immunization the antigenic stimulation continues repeatedly are presented. A warning suggestion is made on monitoring the possible augmented oncogenic susceptibility of vaccinated individuals versus the none vaccinated, to evaluate this potential effect of the vaccine.
\end{abstract}

Keywords: Malaria vaccines; Sporozoite; Sterile immunity; Neoplasm

\section{Introduction}

When Laveran [1] discovered the malaria parasites, more than 80 years had passed since the application of the first vaccine in 1796 by Edward Jenner against the virus of the small pox [2]. On Laveran's time researchers were familiarized with vaccines against virus and bacteria, the proposal of a vaccine against Plasmodium to help in the control and care of malaria, would be of general acceptance, thus with the discovery of the malaria parasites the race for a vaccine had began. Since then, all imaginable techniques and procedures for immunization have been tried with the most diverse immunogenic preparations, blood extracts, extracts of isolated parasite stages, all types of chemically and physically inactivated parasites, isolated and purified parasite proteins, living parasites, death parasite bodies, attenuated live parasites, synthetic antigens, and several added adjuvants among many others.

Results were sometimes promising, sometimes deceptive, most times unpredictable, and most times non repeatable even for the same researcher, at last, after 137 years of research we still have not a successful antimalaric vaccine of general and systematic application. However, among all this massive searching something seems to have been clear, that best immunization had been obtained with whole body and living antigens i.e. reproducing infection itself, and from malaria living vaccines experiences, those prepared from sporozites stage had been the most promising.

\section{The Sporozoite Vaccines}

First experimental vaccination against sporozoites was done by the Sergent brothers in 1910 in a model of bird's malaria with Plasmodium relictum. Isolated sporozoites were attenuated by suspension in saline solution, with no nutrients, for different times, before inoculation into the birds. The Sergent reported protection in $60 \%$ of their birds [3]. Second more successful sporozoites experimental vaccination was done by Ruth Nussenzweig, at the Rockefeller University in 1967, attenuating P. berghei sporozoites by radiation to obtain for the first time sterile immunity against mammalian malaria parasites (mice), with $90 \%$ of protection $[4,5]$.

Probably the third more succesful sporozoites vaccination was done by Meta Roestenberg at the University of Nijmegen, Holland in 2009, immunizing human volunteers with therapeutic levels of chloroquine in their blood by the bite of $P$. falciparum infected mosquitos, achieving 100\% sterile protection [6]. Nussenzweig and Roestenberg works were the foundation for the vaccine development of attenuated, living, purified, and cryopreserve sporozoites going on now in 2017. This vaccine offers $100 \%$ sterile immune protection when applied by intravenous route $[7,8]$.

Experimental Sterile Immunization in Malaria and Cancer Development

Some years ago, we performed a series of experiments to observe phagocytosis of malaria parasites by peritoneal 
macrophages. The experiments consisted in the inoculation of Plasmodium yoelii infected blood into the peritoneum of mice, to take samples of peritoneal exudate, at different times after inoculation to observe if phagocytosis occurred. To trace the parasites, the infected blood to be inoculated was freed of leukocytes by filtration and stained with DAPI then the samples of peritoneal exudates were freshly observed in a glass slide-cover slip preparation, under ultraviolet illumination microscopy. Our first series of experiments were done on healthy no immune mice, in which no phagocytosis was observed. In experiments of a second series, sterile immune mice were included, which we thought will certainly do phagocytosis. Those immune mice included in these experiments were mice inoculated with the lethal strain of Plasmodium yoelii yoelii, for parasite maintenance purpose, that naturally (with no intervention) survive the lethal infection, at random.

Previous studies of these immune mice had determined that these mice acquired a sterile type of immunity and that after each challenge; they exhibit a spurious asymptomatic and transient blood infection with no more that 2 to $3 \%$ of infected erythrocytes that lasted in average 5.5 days. As these immune surviving mice were few and not always available when needed, they were used (intraperitonealy infected) repeatedly in several experiments, giving them a month rest to recover from previous infection. Soon we notice that after several continuous challenges, many of these mice develop neoplastic lesions.

Histopathological studies showed that those tumours developing in sterile immune mice were both malignant and benignant. When we realized the importance of this finding, our initial aims on phagocytosis were changed for the study of neoplasms development associated to malaria infections in sterile immune mice. In more than six years of experiments, we observed the development of about twelve cases of neoplastic growths, most of them malign, including carcinoma, hepatoma, lymphoma and astrocytoma. One of the mice developed a lymphoma that was histopathologically indistinguishable of the human Burkitt's lymphoma. The comparative analysis of the Burkitt's lymphoma-like of the mouse with the human Burkitt's lymphoma of African Children showed us some coincidences, such as that burkitt's lymphoma develops in children at the moment of higher antimalaric immune expression after they have survived malaria infections for 5 to 7 years of their lives, and continue receiving infective mosquito bites (living antigenic stimulation), while in the mouse the neoplastic lesion appear after surviving a lethal infection, that leaded it to a status of sterile immunity and continue receiving living parasites stimulation [9]. It was first suspected that perhaps the neoplasms developed in the mice were probably due to age. To discard spontaneous development of malignancies, ten mice of the same strain used in the experiments were cage, feed and manage under the same conditions as the experimental, and were observed during one and a half years, the result was quite clear, at autopsy no macroscopic or microscopic neoplasms developed in any mice.
Thus neoplastic lesions in the mice were the result of survival to lethal malaria infection, development of sterile immunity and to continuous living antigenic stimulation.

While these experiments were in progress other observation surprise us, and it was the sudden and unexpected death of some of our sterile immune mice, with no signs of disease, without parasites in their blood, with no evident neoplasm development, apparently healthy, and they die from one day to the next. At the moment we have not found what the cause of death in these animals is. One may argue that mouse and human malaria comparison is not valid, because both acquire the infection with different infective stages of the parasite, the sporozoite in humans, coming from an infected mosquito bite, and the blood forms in the mouse, coming directly from another infected mouse. We do not know whether the exoerythrocytic forms or the blood forms are the one associated to the oncogenic stimulation in humans. However, to guide us, one has to remember that an immune state against the parasite seems to be a pre-requisite to induce neoplasms and that in nature exoerytrocytic forms does not elicit an immune state, for this reason we presume that, in nature, Burkitt's lymphoma in humans develops with no immunity against sporozoites, and also, that for the same reason exoerythrocytic forms give pass to the blood forms any time the individual get infected by sporozoites. If experimentally neoplastic development occurs in the mouse lacking of any previous sporozoite infection, it is probable that tumorigenic stimulation be associated to the blood forms present in both hosts. Humans and mice that develop neoplasms, both are immune to their own Plasmodium species, sterile in the mouse and probably also sterile in children, with also the continuous repeated infections as pre-requisite for the eventual development of neoplasms.

\section{Only Burkitt's Lymphoma is Associated with Malaria, or there are Others}

Endemic Burkitt's lymphoma is the only malignancy known to be associated to human malaria in regions of high malaria transmission. Experimental observations showed us that several types of malignancies are developed in $P$. yoelii yoelii sterile immune mice when they are infected repeatedly. Now, one has to ask if that variety of neoplasms that develop in the mice, or similar lesions, doesn't exist in association with human malaria, or they exist but, they have not been yet associated to malaria falciparum or other human Plasmodium species.

\section{The Cryopreserved Sporozoite Vaccine of Plasmodium falciparum}

The prospect of the cryopreserved sporozoite vaccine for the control of P. falciparum has open today an atmosphere of high optimism, in general, present results give substance to expect a great impact on malaria morbidity and mortality around the tropical world. We all hope that the results of its application signify the arrival of a definitively successful malaria vaccine, as 
has been the dreams of malariologists of all times. At the moment, however, the certainty of its efficacy relies on the coming years of application and evaluation, until then, it can be decided if it fill the requisites of the expected vaccine or not.

\section{Conclusion}

Whatever the vaccine results may be, based on the experimental results above commented, it is suggested for ethical and moral considerations, to include in the evaluation of the vaccine the study of morbidity of all type of neoplasm's, autoimmune diseases and inexplicable deaths occurring in vaccinated population versus non vaccinated.

\section{Conflict of Interest}

The authors declare that they have no competing interests regarding to malaria vaccines of any kind. We acknowledge PAPIIT program of DGAPA-Universidad Nacional Autónoma de México for sponsorship of project IN229611

\section{References}

1. Laveran A (1880) Note sur un nouveau parasite trouve dans le sang de plusieurs malade atteins de fiebre palustre. Bull Acad Med Paris 9 $1235-1236$
2. Empson J (1996) Little honoured in his own country: statues in recognition of Edward Jenner MD FRS. J R Soc Med 89: 514-518.

3. Sergent E (1910) Sur l'immunite clans le paludisme des oiseaux. Conservation in vitro des sporozoites de Plasmodium relictum. Immunite reltive obtenue par inoculation de ces sporozoites. Comptes rendues de l'Academie des Sciences Paris 151: 407-409.

4. Nussenzweig RS, Vandenverg J, Most H, Orton C (1967) Protective immunity produced by the injection of x-irradiated sporozoites of Plasmodium berghei. Nature 216: 160-162.

5. Vanderberg JP (2009) Reflections on early malaria vaccine studies, the first successful human malaria vaccination, and beyond. Vaccine 27(1): 2-9.

6. Roestenberg M, MacCall M, Hopman J, Wiersma J, Adrian Jf, et al. (2009) Protection against a malaria challenge by sporozoite inoculation. $\mathrm{N}$ Engl J Med 361: 468-477.

7. Greengood B (2017) Progress with the PfSPZ vaccine for malaria. Lancet Infect Dis 17(5): 463-464.

8. Epstein JE, Paolino KM, Richie TL, Sedegah M, Singer A, et al. (2017) Protection against Plasmodium falciparum malaria by PfSPZ vaccine. JCI Insight 2(1): e89154.

9. Malagón F, González-Angúlo J, Carrasco E, Robert L (2011) Etiopathogenesis of Burkitt's lymphoma: a lesson from a BL-like in CD1 mouse immune to Plasmodium yoelii yoelii. Infect Agent Cancer 6(1): 10.

Your next submission with Juniper Publishers will reach you the below assets

- Quality Editorial service

- Swift Peer Review

- Reprints availability

- E-prints Service

- Manuscript Podcast for convenient understanding

- Global attainment for your research

- Manuscript accessibility in different formats ( Pdf, E-pub, Full Text, Audio)

- Unceasing customer service

Track the below URL for one-step submission https://juniperpublishers.com/online-submission.php 